\title{
The pulmonary histopathologic manifestations of the anti-Jo-1 tRNA synthetase syndrome
}

\author{
Samuel A Yousem ${ }^{1}$, Kevin Gibson ${ }^{2}$, Naftali Kaminski² ${ }^{2}$, Chester V Oddis ${ }^{2}$ and \\ Dana P Ascherman ${ }^{2}$ \\ ${ }^{1}$ Department of Pathology, UPMC Presbyterian Shadyside, Pittsburgh, PA, USA and \\ ${ }^{2}$ Department of Medicine-Dorothy P. and Richard P. Simmons Center for Interstitial Lung Disease and \\ Rheumatology/Clinical Immunology, UPMC Presbyterian Shadyside, Pittsburgh, PA, USA
}

\begin{abstract}
Of the idiopathic inflammatory myopathies, the anti-aminoacyl tRNA synthetase syndrome has the greatest association with interstitial lung disease (ILD). We reviewed 13 open surgical lung biopsies, four autopsies, and three native lungs resected at transplantation, for pulmonary ILD associated with the presence of anti-histidyl tRNA synthetase (anti-Jo-1) autoantibodies. Fifty percent $(N=10)$ of patients presented with an acute decompensation of pulmonary function manifested as diffuse alveolar damage, although in five patients $(25 \%)$ this marked diminution in function was superimposed on an underlying chronic interstitial pneumonia (usual interstitial pneumonia (three); nonspecific interstitial pneumonia(two)). Seven (35\%) patients had usual interstitial pneumonia and two $(10 \%)$ had nonspecific interstitial pneumonia exclusively, whereas one patient presented with an organizing pneumonia (5\%). This study is the first to highlight the high biopsy incidence of diffuse alveolar damage in this patient population both de novo and superimposed on underlying chronic ILD, and also shows that usual interstitial pneumonia remains a significant pattern of interstitial injury in this autoimmune group. On the basis of coexisting patterns of lung injury, this study also suggests that nonspecific interstitial pneumonia in connective tissue disorders may progress over time to a usual interstitial pneumonia pattern of fibrosis, an observation that could be better assessed with future inclusion of autopsy and transplanted native lungs in study groups.
\end{abstract}

Modern Pathology (2010) 23, 874-880; doi:10.1038/modpathol.2010.65; published online 12 March 2010

Keywords: polymyositis; dermatomyositis; Jo-1; antisynthetase; interstitial pneumonia

Idiopathic inflammatory myopathies (IIMs) are a heterogeneous group of systemic inflammatory diseases primarily affecting the skeletal muscles with associated proximal muscle weakness, arthralgias, and increased creatine kinase. Polymyositis and dermatomyositis (PM/DM) comprise the majority of these cases. Within this subgroup, however, are subsets of the IIMs that are characterized by autoantibodies against various aminoacyl-tRNA synthetases. The most common of these is the antiJo-1 antisynthetase syndrome in which anti-histidyl tRNA synthetase (anti-Jo-1) antibodies are accompanied by clinical arthritis, Raynaud's phenomenon, myositis, mechanic's hands, and interstitial lung

Correspondence: Dr SA Yousem, MD, UPMC Presbyterian Shadyside, Department of Pathology, Presbyterian CampusA610, 200 Lothrop Street, Pittsburgh, PA 15213-2582, USA. E-mail: yousemsa@upmc.edu

Received 24 September 2009; revised 11 December 2009; accepted 3 January 2010; published online 12 March 2010 disease (ILD). ${ }^{1-10}$ The anti-Jo-1 tRNA synthetase syndrome has a higher incidence of ILD, relative to classical $\mathrm{PM} / \mathrm{DM}$, approximating $90 \%$ in some series, and the presence of anti-Jo-1 antibodies correlates with poor patient prognosis. ${ }^{1-22}$ In this report, we studied the pulmonary histopathologic changes of a select group of IIM patients with the anti-Jo-1 tRNA synthetase syndrome, generating the first and largest biopsy study focused on this specific group of patients.

\section{Materials and methods}

The patients assessed in this study were derived from the University of Pittsburgh myositis data base and included patients seen at the University of Pittsburgh Medical Center from 1982 through 2009 who consented to registry enrollment. Sera obtained at the time of clinical evaluation were tested for the presence of anti-Jo-1 antibodies using 
immunodiffusion with subsequent confirmation by ELISA. Inclusion in this study required the presence of anti-Jo-1 antibody as well as appropriate clinical data, pulmonary function tests, and imaging studies permitting the ascertainment of ILD at presentation. The diagnosis of ILD required restrictive physiology on pulmonary function testing and an abnormal chest x-ray or an abnormal high resolution computerized tomographic scan (HRCT), showing at least one of the following features preceding the time of surgical lung biopsy: reticulation of fibrosis, traction bronchiectasis, honeycomb change, or ground glass infiltrates. Pathologic assessment was restricted to surgical lung biopsies $(N=13)$, native lungs removed at the time of lung transplantation $(N=3)$, and autopsies $(N=4)$ in which lung disease related to anti-Jo-1 tRNA synthetase syndrome was the cause of death. Clinical information was obtained from patient records and an average of seven hematoxylin and eosin slides (range 3-32) from each case was evaluated. Grocott and acid fast stains for microorganisms were performed on the majority of cases and were negative as were microbiologic cultures for bacteria, mycobacteria, and fungi. Biopsies were classified according to the American Thoracic Society recommendation for pattern classification of ILD including recent recommendations for acute exacerbation of chronic interstitial pneumonias. ${ }^{23-25}$

\section{Results}

The clinicopathologic characteristics of the 20 patients who comprised this study are shown in Tables 1 and 2. Patients were predominantly young women $(\mathrm{F} / \mathrm{M}=4)$ who presented with cough and dyspnea $(N=20 ; 100 \%)$ often with fever $(N=7$; $35 \%)$. The presenting pulmonary manifestations syndromes of this patient population could be classified into three groups: six patients presented with severe rapidly progressive hypoxemia and bilateral widespread alveolar infiltrates with 1 to 2 months of complaints (Group A); nine patients presented with slowly progressive shortness of breath over 6 months to years (Group B); and five patients presented with shortness of breath for several months to years that had abruptly worsened (Group C). Reports of HRCT scans at the time of presentation were reviewed and classified into distinct patterns, which have been shown to correspond with the following pathologic forms of ILD: usual interstitial pneumonia, nonspecific interstitial pneumonia, organizing pneumonia, and acute interstitial pneumonia/diffuse alveolar damage. Of the 15 patients available for study, eight had a usual interstitial pneumonia pattern characterized by bilateral lower lobe reticulations extending to the pleura and accompanied by honeycomb change. Two patients had bilateral patchy areas of ground glass opacities with underlying reticulations and/or
Table 1 Clinical characteristics of anti-Jo-1 antisynthetase syndrome

\begin{tabular}{lc}
\hline $\begin{array}{l}\text { Age (years) } \\
\text { Mean }\end{array}$ & \\
Median & 47.7 \\
Range & 47.0 \\
& $25-65$ \\
Signs and symptoms & \\
Cough & $20(100 \%)$ \\
Dyspnea & $20(100 \%)$ \\
Fever & $7(35 \%)$ \\
Arthritis & $14(70 \%)$ \\
Raynaud's & $8(40 \%)$ \\
Myositis & $20(100 \%)$ \\
& $7(35 \%)$ \\
Smoking history & 50 months (range $1-146$ months) \\
Mean follow-up &
\end{tabular}

Abbreviations: F, female; $\mathrm{M}$, male.

$\mathrm{M}: \mathrm{F}=4: 16$

bronchiectasis corresponding to a nonspecific interstitial pneumonia radiologic pattern, whereas four patients had a rapidly progressive bilateral multilobar consolidation suggestive of the adult respiratory distress syndrome and one patient had patchy peripheral consolidation of the right middle and left lower lobes resembling organizing pneumonia.

The pleuropulmonary histopathologic analysis of the 20 cases with anti-Jo-1 tRNA synthetase syndrome was based on four autopsies, three native lungs removed at the time of unilateral lung transplantation, and 13 surgical biopsies. Seven cases demonstrated a usual interstitial pneumonia pattern of interstitial injury; five cases showed changes of acute and organizing diffuse alveolar damage; and two cases showed features of nonspecific interstitial pneumonia. Interestingly, five additional cases had clinicopathologic features of an acute exacerbation of an underlying chronic interstitial pneumonia with three showing background fibrosis in a usual interstitial pneumonia pattern and two with a background of fibrotic nonspecific interstitial pneumonia. In no case was there evidence of granulomatous disease, vasculitis, alveolar hemorrhage, or malignancy.

A usual interstitial pneumonia pattern of fibrosis was characterized by patchy subpleural and paraseptal interstitial scarring and parenchymal remodeling associated with a variable chronic inflammatory infiltrate and relatively normal intervening parenchyma (Figure 1). Numerous interstitial fibroblastic foci comprised of proliferating fibroblasts and myofibroblasts in a very loose acid mucopolysaccharide rich myxoid background were often seen at the advancing margin of the regions of irreversible scarring (Figure 2). Honeycomb fibrosis was seen in all cases of usual interstitial pneumonia. In contrast, nonspecific interstitial pneumonia patterns of fibrosis were associated septal collagenization in the setting of preserved alveolar architecture 
Table 2 Pathologic diagnosis in anti-Jo-1 antisynthetase syndrome

\begin{tabular}{|c|c|c|c|c|c|}
\hline Case & Age/sex (years) & Clinical group ${ }^{\mathrm{a}}$ & Procedure & Pathologic diagnosis ${ }^{\mathrm{a}}$ & Current status \\
\hline 1 & $40 \mathrm{~F}$ & A & $\mathrm{BX}$ & DAD & DOD, 1 month \\
\hline 2 & $55 \mathrm{~F}$ & A & A & DAD & DOD, 1 month \\
\hline 3 & $42 \mathrm{M}$ & A & $\mathrm{BX}$ & DAD & AWED, 59 months \\
\hline 4 & $62 \mathrm{~F}$ & A & $\mathrm{BX}$ & DAD & AWED, 12 months \\
\hline 5 & $35 \mathrm{~F}$ & A & $\mathrm{BX}$ & DAD & AWED, 22 months \\
\hline 6 & $52 \mathrm{~F}$ & A & $\mathrm{BX}$ & $\mathrm{OP}$ & AWED, 31 months \\
\hline 7 & $57 \mathrm{M}$ & $\mathrm{B}$ & $\mathrm{BX}$ & UIP & Lost to $\mathrm{F} / \mathrm{U}$ \\
\hline 8 & $64 \mathrm{~F}$ & $\mathrm{~B}$ & A & UIP & DOD, 1 months \\
\hline 9 & $35 \mathrm{M}$ & $\mathrm{B}$ & SLTX & UIP & AWED, 48 months \\
\hline 10 & $41 \mathrm{~F}$ & B & SLTX & UIP & AWED, 146 months \\
\hline 11 & $43 \mathrm{~F}$ & B & BX & UIP & AWED, 81 months \\
\hline 12 & $55 \mathrm{M}$ & B & $\mathrm{BX}$ & UIP & AWED, 49 months \\
\hline 13 & $53 \mathrm{~F}$ & B & SLTX & UIP & AWED, 38 months \\
\hline 14 & $40 \mathrm{~F}$ & $\mathrm{~B}$ & $\mathrm{BX}$ & NSIP & AWED, 19 months \\
\hline 15 & $47 \mathrm{~F}$ & $\mathrm{~B}$ & $\mathrm{BX}$ & NSIP & AWED, 14 months \\
\hline 16 & $47 \mathrm{~F}$ & $\mathrm{C}$ & A & NSIP w/acute exacerbation & DOD, 1 month \\
\hline 17 & $25 \mathrm{~F}$ & $\mathrm{C}$ & BX & UIP w/acute exacerbation & DOD, 23 months \\
\hline 18 & $65 \mathrm{~F}$ & $\mathrm{C}$ & $\mathrm{BX}$ & NSIP w/acute exacerbation & AWED, 84 months \\
\hline 19 & $50 \mathrm{~F}$ & $\mathrm{C}$ & A & UIP w/acute exacerbation & DOD, 1 month \\
\hline 20 & $46 \mathrm{~F}$ & $\mathrm{C}$ & $\mathrm{TX}$ & UIP w/acute exacerbation & DOD, 29 months \\
\hline
\end{tabular}

Abbreviations: A, autopsy; AWED, alive with evidence of disease; Bx, surgical lung biopsy; DOD, dead of disease; F, female; F/U, follow-up; $\mathrm{M}$, male; SLTX, single lung transplantation.

${ }^{\mathrm{a}}$ See text for subgroup classification.

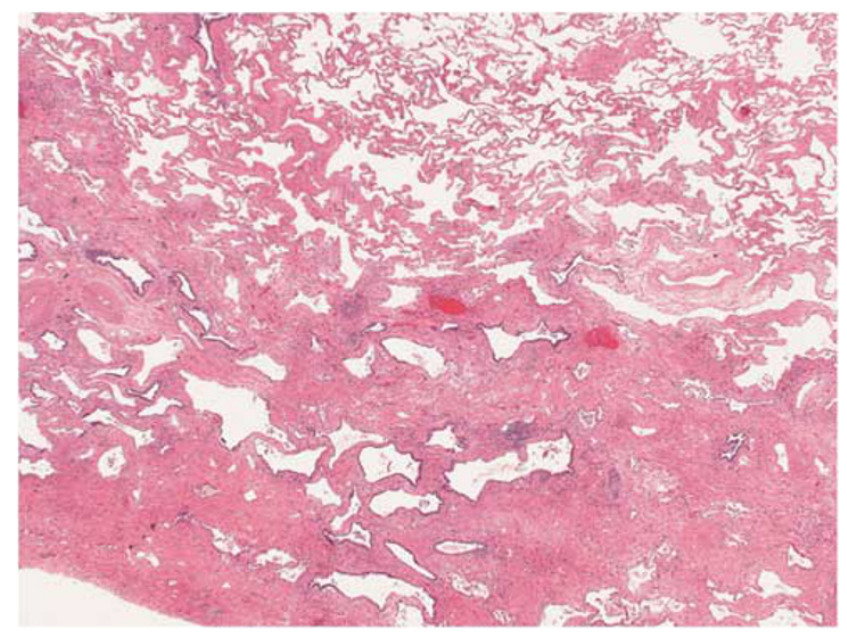

Figure 1 Usual interstitial pneumonia. Usual interstitial pneumonia was characterized by a patchy predominantly subpleural process marked by alternating zones of remodeled fibrotic lung parenchyma and accompanying honeycomb change, associated with other zones with parenchymal scarring and a patchy inflammatory infiltrate with fibroblastic foci present at the advancing interstitial margin.

and were characterized by diffuse involvement of the pulmonary parenchyma by a uniform interstitial thickening of alveolar septae with increased amounts of septal collagen and a mild inflammatory infiltrate consisting of mononuclear cells dominated by lymphocytes and plasma cells (Figures 3 and 4). Marked remodeling of the pulmonary parenchyma was not identified and honeycomb change was absent. A diffuse alveolar damage pattern of injury was characterized by diffuse widespread involve-

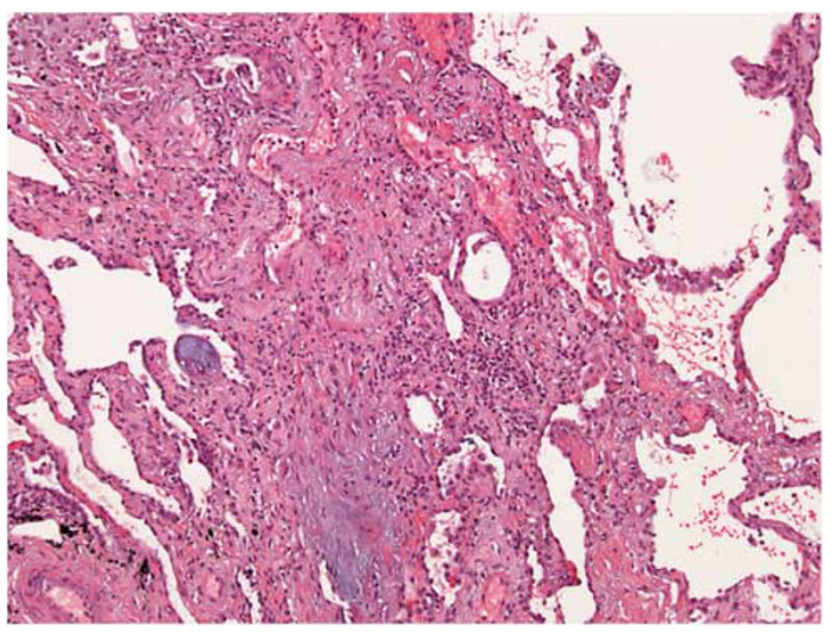

Figure 2 Usual interstitial pneumonia. Fibroblastic foci, appeared as loose basophilic myxoid areas of active interstitial remodeling often occurring at the edge of zones of previously scarred pulmonary parenchyma. Usual interstitial pneumonia had a very sparse chronic inflammatory infiltrate and often was accompanied by areas of mucostasis and bronchiolar metaplasia.

ment of the pulmonary parenchyma and the pulmonary lobule by a pauci-inflammatory process characterized by airspace hyaline membranes, which were often disrupted and incorporated into loose fibromyxoid airspace plugs of granulation tissue, some in variable stages of incorporation into thickened alveolar septa covered by reactive pneumocytes (Figures 5 and 6). This process often was associated with lobular collapse and the absence of histologically normal pulmonary parenchyma. Of 


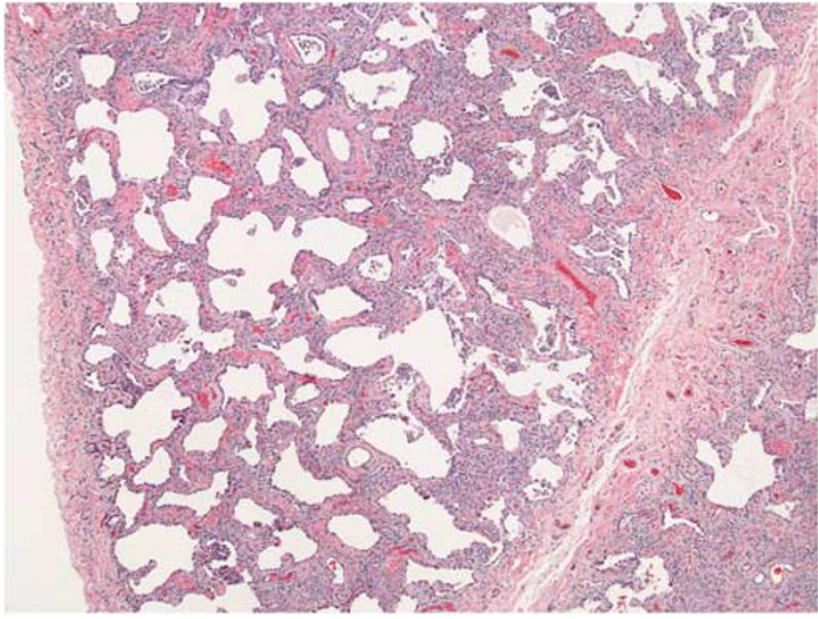

Figure 3 Nonspecific interstitial pneumonia. Nonspecific interstitial pneumonia was characterized by a relatively diffuse inflammatory process with alveolar septal thickening caused by a mononuclear inflammatory infiltrate rich in lymphocytes and plasma cells, with most alveolar septae containing increased amounts of eosinophilic collagen.

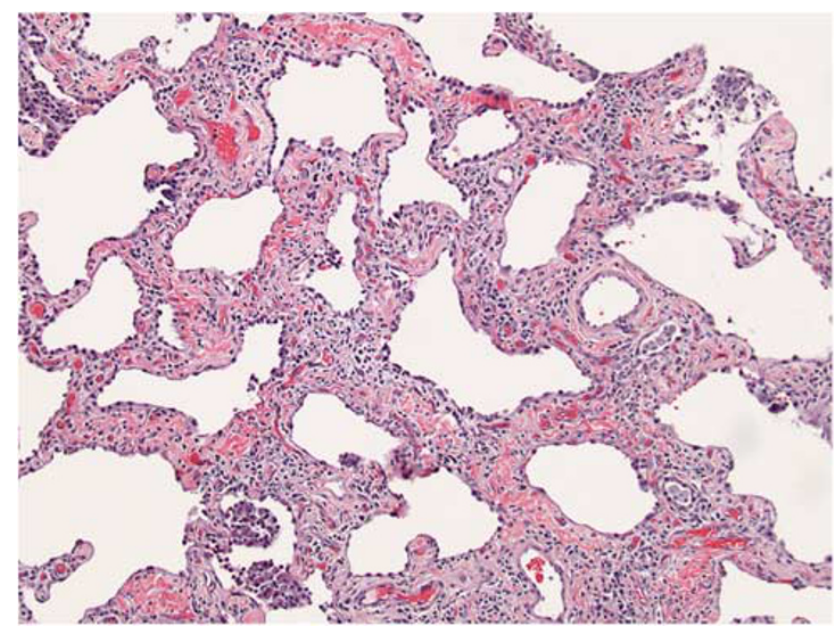

Figure 4 Nonspecific interstitial pneumonia. Higher magnification of alveolar septae in nonspecific interstitial pneumonia showed that most septae were both collagenized by lamellar cords of eosinophilic collagen, often accompanied by chronic inflammatory cell infiltrate in the absence of fibroblastic foci or zones of honeycomb change.

note, this pattern contrasted with an organizing pneumonia pattern of injury where regions of normal lung alternated with other zones where intra-airway and intra-airspace granulation tissue, often in a centrilobular distribution, consolidated the pulmonary parenchyma, and corresponded to an organizing pneumonia pattern of injury. Finally, in cases classified as acute exacerbation of a chronic interstitial pneumonia, a pattern of diffuse alveolar damage was superimposed on a background pulmonary parenchyma showing either diffuse uniform alveolar septal scarring discordant with the age of the acute lung injury, or a pattern of scarring

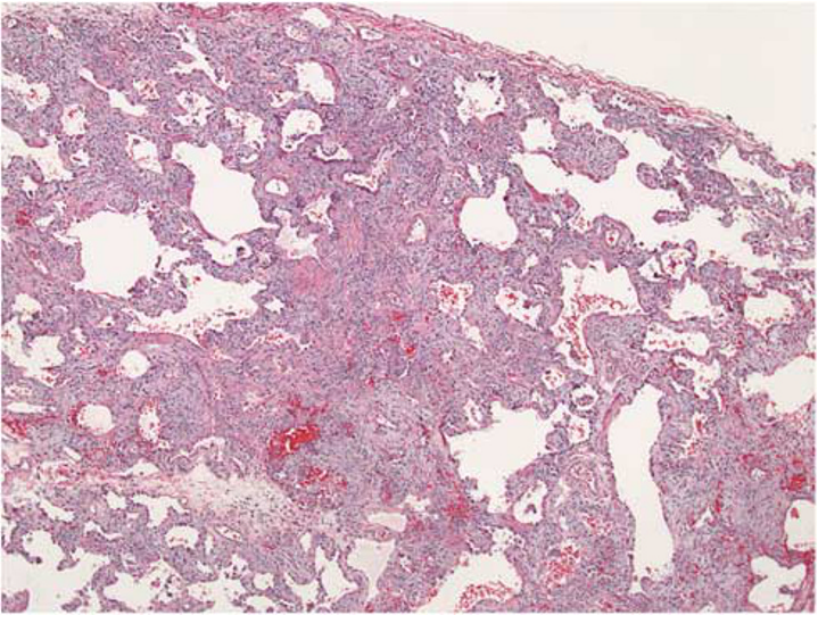

Figure 5 Diffuse alveolar damage. Diffuse alveolar damage manifested as widespread involvement of nearly all alveolar septae by a pauci-inflammatory process characterized by airspace hyaline membranes and granulation tissue, which were variably incorporated into the alveolar septa. Background pulmonary parenchyma appeared preserved without architectural remodeling or distortion.

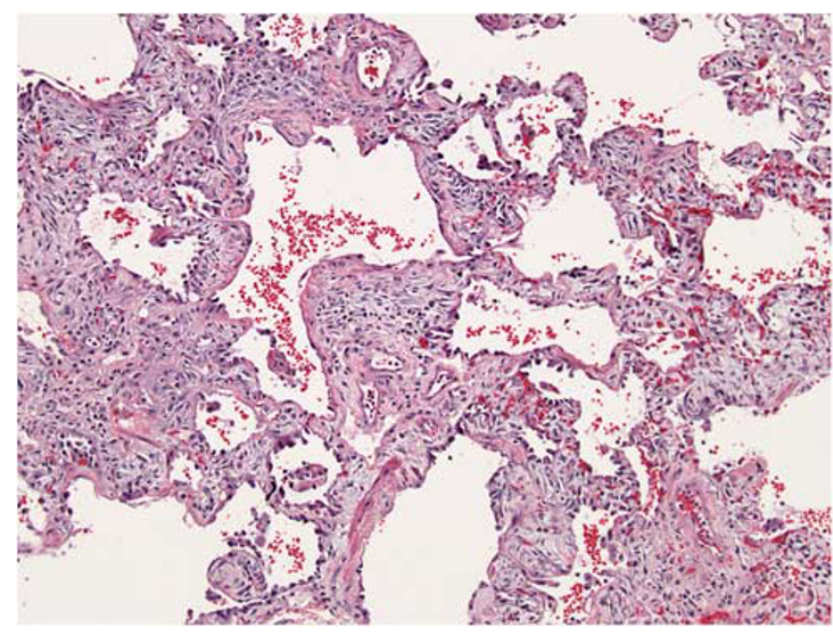

Figure 6 Diffuse alveolar damage. Nearly all alveolar septae were expanded by a loose fibromyxoid connected tissue process associated with activated fibroblasts and myofibroblasts with reactive type 2 pneumocytes coating thickened alveolar septa. In some areas, airspace edema and residual hyaline membranes were identified.

characterized by honeycomb fibrosis in a patchy distribution (Figure 7). The former was classified as a nonspecific interstitial pneumonia pattern of background fibrosis, whereas the latter was classified as the usual interstitial pneumonia pattern of background fibrosis.

\section{Discussion}

Pleuropulmonary manifestations of the PM/DM complex of myopathic autoimmune diseases are multiple ranging from aspiration pneumonia, 


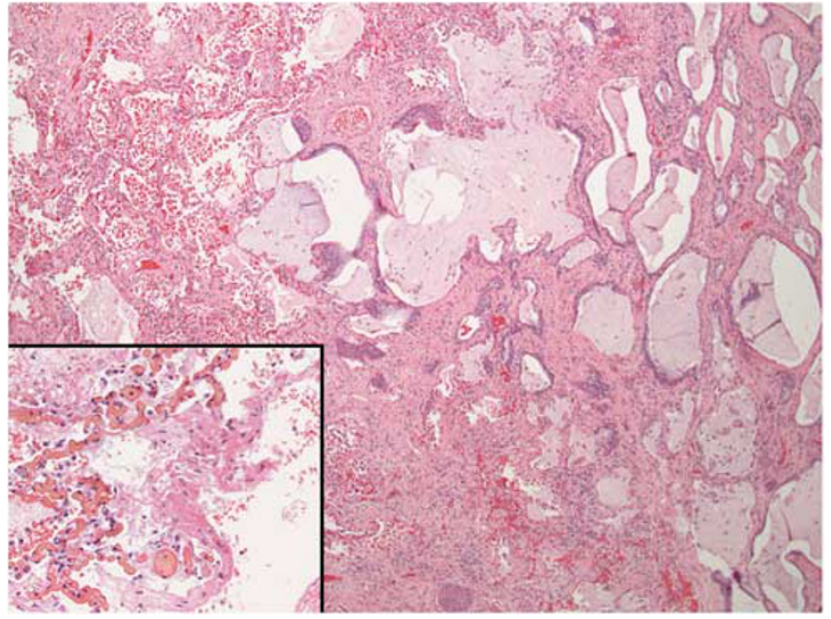

Figure 7 Acute exacerbation, usual interstitial pneumonia. In the setting of marked pulmonary parenchymal scarring and remodeling, the remaining 'normal' lung demonstrated widespread acute lung injury with hyaline membrane formation (insert).

opportunistic infections, drug-induced lung disease, alveolar hemorrhage, vasculitis, and respiratory dysfunction related to disrupted chest wall and diaphragmatic mechanics. ${ }^{15}$ Although such appearances are common, the major morbidity and mortality is tied to ILD, which affects $5-40 \%$ of this patient population. ${ }^{17,19}$ Within this group of PM/DM patients, however, is a subgroup with anti-Jo-1 autoantibodies that stand out because of their remarkable association with pulmonary function and chest radiographic abnormalities occurring in up to $90 \%$ of cases. ${ }^{12,15-17,19,21,22}$ This study represents the largest and most complete study of surgical lung biopsies in this unique patient subset and provides some perspectives on the clinical course of this disease given the inclusion of late stage cases derived from lung transplantation and autopsy programs.

Historically, studies of PM/DM patients with ILD manifested primarily as usual interstitial pneumonia, organizing pneumonia, and diffuse alveolar damage. ${ }^{4,20}$ With the recognition of nonspecific interstitial pneumonia as a distinct histopathologic entity, ${ }^{26}$ more recent studies have reported the preponderance of nonspecific interstitial pneumonia in the PM/DM patient population and the relative infrequency of other patterns of lung injury. ${ }^{11,12,21,27}$ Yet, this study of the anti-Jo-1 antibody positive subset of patients highlights several current differences from traditional PM/DM that warrant discussion. First, a significant manifestation of Jo-1 disease is an acute decompensation of lung function that corresponds to widespread acute lung injury $(N=5 ; 20 \%)$ and has a radiographic pattern of bilateral widespread ground glass changes that if devoid of clinical information resembles a form of nonspecific interstitial pneumonia. Particularly, in its late healing phase, diffuse alveolar damage has morphologic similarities to nonspecific interstitial pneumonia as noted by Katzenstein and Fiorelli ${ }^{26}$ that may, in some studies, be responsible for the reported increased incidence in autoimmune diseases. Review of multiple studies indicates that this histologic pattern of ILD corresponds to the acute clinical presentations that often result in death. ${ }^{11,17,20,21,27}$ What our study uniquely demonstrates is a second manifestation of diffuse alveolar damage, specifically that it is often superimposed on underlying chronic fibrosing lung disease, particularly usual interstitial pneumonia and fibrotic forms of nonspecific interstitial pneumonia $(N=5 ; 20 \%)$. Such acute exacerbations have been recently recognized in the idiopathic chronic interstitial pneumonias and are first documented here in our cohort of anti-Jo-1 disease ${ }^{24,25}$ that includes an important array of transplant native lungs and autopsy material. This finding also confirms observations of Tansey et $a l^{27}$ that multiple patterns of lung injury often occur in autoimmune lung disease.

The relatively high percentage of usual interstitial pneumonia patients in our anti-Jo-1 group when compared with other recent $\mathrm{PM} / \mathrm{DM}$ studies is also noteworthy. Although usual interstitial pneumonia may simply be more prevalent in the anti-Jo-1 subset de novo, ${ }^{28}$ this finding may relate to the inclusion of material from three lung transplant recipients and four autopsy cases. Interestingly, as noted in the literature, there is a subgroup of 'typical' nonspecific interstitial pneumonia patients who on longitudinal radiographic study progress to a pattern of lung damage resembling usual interstitial pneumonia who are captured only by studies containing autopsy and lung transplant cases. ${ }^{29,30}$ Coupled with our observation of a high percentage of usual interstitial pneumonia patterns of fibrosis, such clinical data suggests that nonspecific interstitial pneumonia in the anti-Jo-1 population could evolve over time into a usual interstitial pneumonia pattern of fibrosis as well as traditional fibrotic forms of nonspecific interstitial pneumonia. This possibility should be addressed in future autopsy studies of nonspecific interstitial pneumonia. Another observation in this study of native resected lungs confirms Katzenstein et al's ${ }^{31}$ observations that in usual interstitial pneumonia, focal areas of nonspecific interstitial pneumonia and DIP are common. Our native lungs with usual interstitial pneumonia showed broad expanses of nonspecific interstitial pneumonia like change supporting the evolutionary concept of usual interstitial pneumonia development through a nonspecific interstitial pneumonia precursor. Another caveat in interpreting other studies reporting a high incidence of nonspecific interstitial pneumonia is that many of these studies include transbronchial biopsies that could easily distort the incidence of reported histopathologic subtypes such as usual interstitial pneumonia. Certainly, the discrepancies in the percentages of usual interstitial pneumonia of our study relative to other studies, encourages longitudinal investigations of nonspecific interstitial pneumonia patients to autopsy or native 
lungs resected at transplantation, and suggests that patients with connective tissue disorders who are monitored for ILD from an early stage will be a study group who could provide information on the biologic progression of chronic ILD.

In patients with $\mathrm{PM} / \mathrm{DM}$, predictors of ILD include the presence of arthritis, older age, and anti-Jo-1 antibodies. .,8,10,11,13,17,19,21 $^{\text {Morbidity in }}$ both PM/DM and the anti-Jo-1 syndrome is related to acute respiratory decompensation at presentation and our study confirms this association and points out that the morphologic equivalent may be diffuse alveolar damage alone or acute lung injury superimposed on underlying fibrotic interstitial pneumonias such as usual interstitial pneumonia and fibrotic nonspecific interstitial pneumonia. ${ }^{5,8,17,19,21}$

The limitations to this study are notable. First, this is a retrospective study, where clinical information, largely based on the referral nature of the patient population to the University of Pittsburgh, is potentially restricted. This bias limited the ability to perform and study sequential HRCT scans that could document the natural history of chronic fibrosing lung disease from very early time points in this patient population. Second, autopsies were not performed on all patients creating a gap in our ability to fully define the natural history of this subgroup. Finally, referral bias could certainly impact the distribution of histopathologic subtypes observed in our cohort based on selection of the most critically ill patients for biopsy procedures often performed at the time of lung transplantation or death.

In summary, this study represents the first and largest study of a specific subgroup of IM patients with the anti-Jo-1 tRNA synthetase syndrome who have had extensive lung parenchymal evaluations. This analysis highlights that a significant portion of these patients present with acute respiratory decompensation manifesting as pure diffuse alveolar damage or diffuse alveolar damage superimposed on underlying chronic symptomatic or asymptomatic fibrosing lung disease. This study underscores the concept that although nonspecific interstitial pneumonia characterizes a significant subset of anitJo-1 antibody positive respiratory disease, usual interstitial pneumonia represents the most common chronic pattern seen at the time of biopsy, lung transplantation, or autopsy. Overall, this insight pertaining to the natural history of anti-Jo-1 antibody positive ILD underscores the need for more systematic functional, radiographic, and histopathologic tracking that will be necessary to promote earlier therapeutic intervention and improve clinical outcome in this unique group of patients.

\section{Acknowledgement}

We appreciate the continued and irreplaceable efforts of Diana Winters in producing this manuscript.

\section{Disclosure/conflict of interest}

The authors declare no conflict of interest.

\section{References}

1 Friedman AW, Targoff IN, Arnett FC. Interstitial lung disease with autoantibodies against aminoacyl-tRNA synthetases in the absence of clinically apparent myositis. Semin Arthritis Rheum 1996;26:459-467.

2 Gomard-Mennesson E, Fabien N, Cordier JF, et al. Clinical significance of anti-histidyl-tRNA synthetase (Jo1) autoantibodies. Ann N Y Acad Sci 2007;1109: 414-420.

3 Hengstman GJ. Advances in the immunopathophysiology of the idiopathic inflammatory myopathies: not as simple as suspected. Curr Rheumatol Rep 2007;9: 280-285.

4 Ioannou Y, Sultan S, Isenberg DA. Myositis overlap syndromes. Curr Opin Rheumatol 1999;11:468-474.

5 Kang EH, Lee EB, Shin KC, et al. Interstitial lung disease in patients with polymyositis, dermatomyositis and amyopathic dermatomyositis. Rheumatology (Oxford) 2005;44:1282-1286.

6 Marguerie C, Bunn CC, Beynon HL, et al. Polymyositis, pulmonary fibrosis and autoantibodies to aminoacyltRNA synthetase enzymes. Q J Med 1990;77: 1019-1038.

7 Miller FW. Myositis-specific autoantibodies. Touchstones for understanding the inflammatory myopathies. JAMA 1993;270:1846-1849.

8 Spath M, Schroder M, Schlotter-Weigel B, et al. The long-term outcome of anti-Jo-1-positive inflammatory myopathies. J Neurol 2004;251:859-864.

9 Targoff IN. Myositis specific autoantibodies. Curr Rheumatol Rep 2006;8:196-203.

10 Yoshifuji H, Fujii T, Kobayashi S, et al. Anti-aminoacyl-tRNA synthetase antibodies in clinical course prediction of interstitial lung disease complicated with idiopathic inflammatory myopathies. Autoimmunity 2006;39:233-241.

11 Chen IJ, Jan Wu YJ, Lin CW, et al. Interstitial lung disease in polymyositis and dermatomyositis. Clin Rheumatol 2009;28:639-646.

12 Douglas WW, Tazelaar HD, Hartman TE, et al. Polymyositis-dermatomyositis-associated interstitial lung disease. Am J Respir Crit Care Med 2001;164: $1182-1185$.

13 Fathi M, Dastmalchi M, Rasmussen E, et al. Interstitial lung disease, a common manifestation of newly diagnosed polymyositis and dermatomyositis. Ann Rheum Dis 2004;63:297-301.

14 Fathi M, Lundberg IE. Interstitial lung disease in polymyositis and dermatomyositis. Curr Opin Rheumatol 2005;17:701-706.

15 Fathi M, Lundberg IE, Tornling G. Pulmonary complications of polymyositis and dermatomyositis. Semin Respir Crit Care Med 2007;28:451-458.

16 Fujita J, Ohtsuki Y, Yoshinouchi T, et al. Idiopathic non-specific interstitial pneumonia: as an 'autoimmune interstitial pneumonia'. Respir Med 2005;99: 234-240.

17 Marie I, Hachulla E, Cherin P, et al. Interstitial lung disease in polymyositis and dermatomyositis. Arthritis Rheum 2002;47:614-622. 
18 Plastiras SC, Soliotis FC, Vlachoyiannopoulos P, et al. Interstitial lung disease in a patient with antisynthetase syndrome and no myositis. Clin Rheumatol 2007;26:108-111.

19 Schnabel A, Reuter M, Biederer J, et al. Interstitial lung disease in polymyositis and dermatomyositis: clinical course and response to treatment. Semin Arthritis Rheum 2003;32:273-284.

20 Tazelaar HD, Viggiano RW, Pickersgill J, et al. Interstitial lung disease in polymyositis and dermatomyositis. Clinical features and prognosis as correlated with histologic findings. Am Rev Respir Dis 1990;141: 727-733.

21 Tillie-Leblond I, Wislez M, Valeyre D, et al. Interstitial lung disease and anti-Jo-1 antibodies: difference between acute and gradual onset. Thorax 2008;63: 53-59.

22 Vancsa A, Csipo I, Nemeth J, et al. Characteristics of interstitial lung disease in SS-A positive/Jo-1 positive inflammatory myopathy patients. Rheumatol Int 2009;29:989-994.

23 American Thoracic Society/European Respiratory Society International Multidisciplinary Consensus Classification of the Idiopathic Interstitial Pneumonias. This joint statement of the American Thoracic Society (ATS), and the European Respiratory Society (ERS) was adopted by the ATS board of directors, June 2001 and by the ERS Executive Committee, June 2001. Am J Respir Crit Care Med 2002;165:277-304.
24 Collard HR, Moore BB, Flaherty KR, et al. Acute exacerbations of idiopathic pulmonary fibrosis. Am J Respir Crit Care Med 2007;176:636-643.

25 Travis WD, Hunninghake G, King Jr TE, et al. Idiopathic nonspecific interstitial pneumonia: report of an American Thoracic Society project. Am J Respir Crit Care Med 2008;177:1338-1347.

26 Katzenstein AL, Fiorelli RF. Nonspecific interstitial pneumonia/fibrosis. Histologic features and clinical significance. Am J Surg Pathol 1994;18:136-147.

27 Tansey D, Wells AU, Colby TV, et al. Variations in histological patterns of interstitial pneumonia between connective tissue disorders and their relationship to prognosis. Histopathology 2004;44: $585-596$.

28 Ascherman DP. The role of Jo-1 in the immunopathogenesis of polymyositis: current hypotheses. Curr Rheumatol Rep 2003;5:425-430.

29 Marten K, Milne D, Antoniou KM, et al. Non-specific interstitial pneumonia in cigarette smokers: a CT study. Eur Radiol 2009;19:1679-1685.

30 Silva CI, Muller NL, Hansell DM, et al. Nonspecific interstitial pneumonia and idiopathic pulmonary fibrosis: changes in pattern and distribution of disease over time. Radiology 2008;247:251-259.

31 Katzenstein AL, Zisman DA, Litzky LA, et al. Usual interstitial pneumonia: histologic study of biopsy and explant specimens. Am J Surg Pathol 2002;26: 1567-1577. 